\title{
NONISOMORPHIC ALGEBRAIC STRUCTURES ON SMOOTH MANIFOLDS
}

\author{
J. BOCHNAK AND W. KUCHARZ
}

(Communicated by William C. Waterhouse)

\begin{abstract}
Given a compact smooth hypersurface $M$ in $\mathbf{R}^{n+1}$, we construct a family $\left\{X_{k}\right\}, k=1,2, \ldots$, of nonsingular real algebraic subsets of $\mathbf{R}^{n+1}$ such that each $X_{k}$ is isotopic to $M$ but, for $k \neq l, X_{k}$ and $X_{l}$ are not birationally equivalent.
\end{abstract}

Let $X \subset \mathbf{R}^{n}$ and $Y \subset \mathbf{R}^{p}$ be locally closed sets in the Zariski topology. A map $f: X \rightarrow Y$ is said to be regular if there exist polynomials $f_{i}, g_{i}$ in $\mathbf{R}\left[x_{1}, \ldots, x_{n}\right]$, $g_{i}^{-1}(0) \cap X=\varnothing, i=1, \ldots, p$, such that $f(x)=\left(f_{1}(x) / g_{1}(x), \ldots, f_{p}(x) / g_{p}(x)\right)$ for all $x$ in $X$. A bijective map $f: X \rightarrow Y$ is called a regular isomorphism if $f$ and $f^{-1}$ are regular maps; $X$ and $Y$ are called birationally equivalent if there exist Zariski open and dense subsets $U$ and $V$ of $X$ and $Y$, respectively, which are regularly isomorphic.

Let $M$ be a compact smooth (i.e., $C^{\infty}$ ) manifold and let $\Omega_{\mathrm{alg}}(M)$ be the set of all equivalence classes of pairs $(X, \varphi)$, where $X$ is a nonsingular real algebraic set, $\varphi: X \rightarrow M$ is a smooth diffeomorphism, and two pairs $\left(X_{1}, \varphi_{1}\right),\left(X_{2}, \varphi_{2}\right)$ are considered to be equivalent if there exists a regular isomorphism $\gamma: X_{1} \rightarrow X_{2}$ satisfying $\varphi_{1}=\varphi_{2} \circ \gamma$. Thus $\Omega_{\mathrm{alg}}(M)$, originally introduced by Akbulut and King $[\mathbf{1}]$, is the set of distinct algebraic structures on $M$ (the moduli space of $M$ ).

It is well known that if we replace in the above definition of $\Omega_{\mathrm{alg}}(M)$ the real data by the complex ones, i.e., if $M$ is a compact complex manifold, $X$ a complex projective algebraic set, $\varphi$ a biholomorphism, and $\gamma$ a complex regular isomorphism, then the corresponding space $\Omega_{\mathrm{alg}}(M, \mathbf{C})$ is either empty or has exactly one element (cf. [5, p. 68]). The situation is quite different in the real case. The space $\Omega_{\mathrm{alg}}(M)$ is never empty by a well-known theorem of Nash-Tognoli [1]. Akbulut and King $[1,2]$ described how to construct smooth manifolds $M$ with $\Omega_{\mathrm{alg}}(M)$ containing at least two elements, but their method does not allow us to prove that $\Omega_{\text {alg }}(M)$ is infinite. On the other hand one easily shows that $\Omega_{\text {alg }}\left(S^{n}\right)$, where $S^{n}$ is the unit $n$-dimensional sphere, is an infinite set provided $n \geq 1$. Indeed, for each integer $k$, $k \geq(n+1) / 2$, consider the complex algebraic projective set $V_{k}=\left\{\left[z_{1}, \ldots, z_{n+2}\right] \in\right.$ $\left.\mathbf{C} P^{n+1} \mid z_{1}^{2 k}+\cdots+z_{n+1}^{2 k}=z_{n+2}^{2 k}\right\}$. Clearly, $V_{k}$ is nonsingular and its arithmetic genus $p_{a}\left(V_{k}\right)$ is equal to $\left(\begin{array}{c}2 k-1 \\ n+1\end{array}\right)\left[4\right.$, p. 54]. Thus if $k \neq l$, then $p_{a}\left(V_{k}\right) \neq p_{a}\left(V_{l}\right)$ and hence $V_{k}$ and $V_{l}$ are not birationally equivalent as complex varieties (cf. [4, p. 431]). Now identify $\mathbf{R}^{n+1}$ with a subset of $\mathbf{C} P^{n+1}$ using the map $\left(x_{1}, \ldots, x_{n+1}\right) \rightarrow$ $\left[x_{1}, \ldots, x_{n+1}, 1\right]$. Note that $S^{n}(k)=V_{k} \cap \mathbf{R}^{n+1}=\left\{\left(x_{1}, \ldots, x_{n+1}\right) \in \mathbf{R}^{n+1} \mid x_{1}^{2 k}+\right.$ $\left.\cdots+x_{n+1}^{2 k}=1\right\}$ is a nonsingular real algebraic set diffeomorphic to $S^{n}$. Moreover,

Received by the editors February 10, 1986 and, in revised form, September 9, 1986.

1980 Mathematics Subject Classification (1985 Revision). Primary 14E05, 14M99. 
the Zariski closure of $S^{n}(k)$ in $\mathbf{C} P^{n+1}$ coincides with $V_{k}$. It follows that if $S^{n}(k)$ and $S^{n}(l)$ are birationally equivalent, then so are $V_{k}$ and $V_{l}$. Hence $\Omega_{\text {alg }}\left(S^{n}\right)$ is an infinite set.

The following conjecture seems to be reasonable.

CONJECTURE. For every compact smooth manifold $M$, the set $\Omega_{\mathrm{alg}}(M)$ is infinite.

We shall prove this conjecture in some special cases.

THEOREM. Let $M$ be a smooth compact hypersurface in $\mathbf{R}^{n+1}, n \geq 1$. Then there exists a family $\left\{X_{k}\right\}, k=1,2, \ldots$, of nonsingular algebraic subsets of $\mathbf{R}^{n+1}$ such that $X_{k}$ is isotopic to $M$ and for $k \neq l, X_{k}$ and $X_{l}$ are not birationally equivalent. In particular, the set $\Omega_{\mathrm{alg}}(M)$ is infinite.

ProOF. The proof is a refinement of the idea applied above for $S^{n}$. We identify $\mathbf{R}^{n+1}$ with a subset of the real projective space $\mathbf{R} P^{n+1}$ via the map $\left(x_{1}, \ldots, x_{n+1}\right) \rightarrow\left[x_{1}, \ldots, x_{n+1}, 1\right]$. Since $M$ bounds in $\mathbf{R} P^{n+1}$, there exists a smooth function $f: \mathbf{R} P^{n+1} \rightarrow \mathbf{R}$ with 0 in $\mathbf{R}$ being its regular value and $M=$ $f^{-1}(0)$. Let $p: S^{n+1} \rightarrow \mathbf{R} P^{n+1}$ be the standard projection. Fix a convex neighborhood $\mathcal{U}$ of $f \circ p$, in the space of all smooth functions from $S^{n+1}$ to $\mathbf{R}$ equipped with the $C^{\infty}$ topology, such that for each $g$ in $U$ the function $S^{n+1} \rightarrow \mathbf{R}$, defined by $x \rightarrow g(-x)$ for $x$ in $S^{n+1}$, also belongs to $U$ and the set

$$
H(g)=\left\{[x] \in \mathbf{R} P^{n+1} \mid \frac{1}{2}(g(x /\|x\|)+g(-x /\|x\|))=0\right\}
$$

is a smooth submanifold of $\mathbf{R}^{n+1}$ isotopic to $M$ in $\mathbf{R}^{n+1}$. By the Weierstrass approximation theorem, there exists a polynomial map $\varphi: \mathbf{R}^{n+2} \rightarrow \mathbf{R}$ whose restriction to $S^{n+1}$ belongs to $\mathcal{U}$. Let $\psi(x)=\frac{1}{2}(\varphi(x)+\varphi(-x))$ for $x$ in $\mathbf{R}^{n+1}$ and let $d$ be the degree of $\psi$. Obviously, $d$ is even.

Now for each positive integer $k$, define a function $f_{k}: \mathbf{R}^{n+2} \rightarrow \mathbf{R}$ by

$$
f_{k}(x)= \begin{cases}\|x\|^{d+2(k+d)} \psi(x /\|x\|) & \text { for } x \in \mathbf{R}^{n+2} \backslash\{0\}, \\ 0 & \text { for } x=0 .\end{cases}
$$

Note that $f_{k}$ is a homogeneous polynomial of even degree $d+2(k+d)$. By a small perturbation of $f_{k}$ in the space of all homogeneous polynomials of degree $d+2(k+d)$, one can obtain a homogeneous polynomial $g_{k}: \mathbf{R}^{n+2} \rightarrow \mathbf{R}$ of degree $d+2(k+d)$ such that the restriction of $g_{k}$ to $S^{n+1}$ belongs to $U$ and 0 in the complex space $\mathbf{C}^{n+2}$ is the only critical point of the complexification $q_{k, \mathbf{C}}$ of $g_{k}$. This is possible since in the vector space of all homogeneous real polynomials in $n+1$ variables of degree at least 2 , the set of polynomials having 0 in $\mathbf{C}^{n+2}$ as the only critical point is dense (cf. for instance [3, proof of Proposition 1]). By construction, $g_{k}(x)=g_{k}(-x)$, the degree of $g_{k}$ being even. It follows that

$$
X_{k}=\left\{[x] \in \mathbf{R} P^{n+1} \mid g_{k}(x)=0\right\}=H\left(g_{k} \mid S^{n+1}\right)
$$

is a nonsingular algebraic subset of $\mathbf{R}^{n+1}$ which is isotopic to $M$ in $\mathbf{R}^{n+1}$. The Zariski (complex) closure $X_{k, \mathbf{C}}$ of $X_{k}$ in the complex projective space $\mathbf{C} P^{n+1}$ is a nonsingular complex projective hypersurface whose homogeneous ideal is generated by $g_{k, \mathbf{C}}$. Therefore the arithmetic genus $p_{a}\left(X_{k, \mathbf{C}}\right)$ of $X_{k, \mathbf{C}}$ is equal to $\left(\begin{array}{c}d+2(k+n)-1 \\ n+1\end{array}\right)$ (cf. [4, p. 54]). If $X_{k}$ and $X_{l}$ are birationally equivalent, then so are $X_{k, \mathrm{C}}$ and $X_{l, \mathrm{C}}$ (as complex varieties) and hence $p_{a}\left(X_{k, \mathrm{C}}\right)=p_{a}\left(X_{l, \mathrm{C}}\right)$ (cf. [4, p. 413, Remark 5.6.1]). Since $d+2(k+n) \geq n+2$, the last equality implies $k=l$. 
COROLLARY. Let $M$ be one of the smooth manifolds lisfted below:

(i) $M$ is the connected sum of a compact smooth hypersurface $N$ in $\mathbf{R}^{n+1}$ and $k$ copies of the projective space $\mathbf{R} P^{n}, n \geq 1$.

(ii) $M$ is a compact surface.

Then $\Omega_{\mathrm{alg}}(M)$ is an infinite set.

ProOF. (i) First observe that if $X_{i}$ is a nonsingular real algebraic set, $Y_{i}$ an algebraic subset of $X_{i}$ and $B\left(X_{i}, Y_{i}\right)$ the algebraic blowing up of $X_{i}$ along $Y_{i}, i=$ 1,2 , then $X_{1}$ is birationally equivalent to $X_{2}$ if and only if $B\left(X_{1}, Y_{1}\right)$ is birationally equivalent to $B\left(X_{2}, Y_{2}\right)$.

Now if $X$ is a nonsingular real algebraic set diffeomorphic to $N$, then by blowing up $k$ points in $X$, one obtains a nonsingular real algebraic set diffeomorphic to the connected sum of $N$ and $k$ copies of $\mathbf{R} P^{n}$. Hence (i) follows from the theorem.

(ii) is a particular case of the theorem if $M$ is orientable and a particular case of (i) if $M$ is nonorientable.

\section{REFERENCES}

1. S. Akbulut and H. King, The topology of real algebraic sets, Enseign. Math. 29 (1983), 221-261.

2. __ Submanifolds and homology of nonsingular real algebraic varieties, Amer. J. Math. 107 (1985), 45-83.

3. J. Bochnak, Relèvement des jets, Lecture Notes in Math., vol. 275 Springer-Verlag, Berlin and New York, 1972, pp. 106-118.

4. R. Hartshorne, Algebraic geometry, Springer-Verlag, Berlin and New York, 1977.

5. D. Mumford, Algebraic geometry. I, Springer-Verlag, Berlin and New York, 1978.

Department of Mathematics, VRije Universiteit, P.O. BoX 7161,1007 MC AMSTERDAM, THE NETHERLANDS

Department of Mathematics and Statistics, University of New Mexico, AlbUQUeRQUe, NEW MEXICO 87131 